\title{
Construction of a cysteine protease deficient Bombyx mori multiple nucleopolyhedrovirus bacmid and its application to improve expression of a fusion protein
}

\author{
Masato Hiyoshi, ${ }^{1}$ Ayano Kageshima, ${ }^{1}$ Tatsuya Kato, ${ }^{1}$ and Enoch Y. Park ${ }^{1,2 *}$ \\ Laboratory of Biotechnology, ${ }^{1}$ Department of Applied Biological Chemistry, \\ ${ }^{2}$ Integrated Bioscience Section, Graduate School of Science and Technology, \\ Shizuoka University, 836 Ohya, Suruga-ku, Shizuoka 422-8529, Japan
}

Abbreviations: Bacmid, baculovirus shuttle vector; BmMNPV, Bombyx mori multiple nucleopolyhedrovirus; bx, signal sequence from Bombyx mori bombyxin; CPD-BmMNPV, cysteine protease-deleted $\mathrm{BmMNPV}$; $\mathrm{GFP}_{\mathrm{uv}}$, green fluorescent protein when excited with long-wave UV light; GGT2, $\mathrm{GFP}_{\mathrm{uv}}-\beta 3 \mathrm{GnT} 2$ fusion protein; PCR, polymerase chain reaction; $v$-cath, papain-type cysteine protease with cathepsin L-like characteristics; $\beta 3 \mathrm{GnT} 2$,

$\beta 1,3-N$-acetylglucosaminyltransferase 2

*Corresponding author. Tel. \& Fax: +81-54-228-4887

E-mail: yspark@agr.shizuoka.ac.jp (E.Y. Park) 


\section{Abstract}

The bacmid system of BmMNPV with cysteine protease gene deletion

3 (CPD-BmMNPV bacmid) was constructed using the lambda recombination system. The

4 protease activities of Bombyx mori cells and silkworm larvae infected with this

5 CPD-BmMNPV bacmid were reduced by $94 \%$ and $85 \%$, respectively. By using this

6 system, a GFP ${ }_{\mathrm{uv}}-\beta 1,3-N$-acetylglucosaminyltransferase $2\left(\mathrm{GFP}_{\mathrm{uv}}-\beta 3 \mathrm{GnT} 2\right)$ fusion

7 protein was successfully expressed in silkworm larvae with less protein degradation and

8 without larvae liquefaction; $\beta 3 \mathrm{GnT}$ activity improved $30 \%$. This CPD-BmMNPV

9 bacmid system provides rapid protein production in silkworms and can be used for the

10 production of recombinant eukaryotic proteins without proteolytic degradation.

11

12 Keywords: Bacmid; Bombyx mori multiple nucleopolyhedrovirus; Cysteine protease;

13 Expression; Silkworm

14 
2

\section{Introduction}

The baculovirus expression system is a useful tool for the efficient production of eukaryotic proteins that require correct folding and posttranslational modification such as signal peptide processing (Davis et al., 1992), phosphorylation (Hericourt et al., 2000) and glycosylation (James et al., 1995). However, Autographa californica multiple nucleopolyhedrovirus has a papain-type cysteine protease with cathepsin L-like characteristics (v-cath) (Slack et al., 1995), which plays an important role in the degradation of host tissues during virus infection. Slack et al. (1995) reported that $\mathrm{v}$-cath has $35.5 \mathrm{kDa}$ and $32 \mathrm{kDa}$ precursor forms that are processed to a $27.5 \mathrm{kDa}$ mature form.

When insect cells are infected with baculoviruses, v-cath accumulates as a propeptide, and the death of infected cells provokes v-cath activation (Hom et al., 2002). This v-cath protease causes the degradation of expressed recombinant proteins, which hinders their efficient production in baculovirus expression systems (Yamada et al., 1990; Pyle et al., 1995; Pham et al., 1999). Kato et al. (2004) expressed a human glycoprotein, $\beta-1,3-N$-acetylglucosaminyltransferase-2 ( $\beta 3 \mathrm{GnT} 2)$ fused with $\mathrm{GFP}_{\mathrm{uv}}$ in Sf9 and Tn-5B1-4 cells using a baculovirus expression system, but $\beta 3 \mathrm{GnT} 2$ activity decreased markedly at 3 days postinjection owing to the presence of $\mathrm{v}$-cath protease.

This suggests that the degradation of recombinant proteins due to v-cath protease is a critical drawback in the expression of glycoproteins using a baculovirus expression 
1

2

3

4

5

6

7

system.

To prevent the proteolytic degradation of expressed proteins, Monsma and Scott (1997) generated BacVector-3000, in which the v-cath protease gene was deleted from Autographa californica multiple nucleopolyhedrovirus, and reported that the degradation of recombinant proteins was markedly reduced.

Protein expression levels using silkworms or pupae are 10- to 100-fold higher than those using Bombyx mori cells or conventional insect cells, indicating that the silkworm or its pupa is one of the most suitable systems for large-scale production of eukaryotic proteins. Recently, Motohashi et al. (2005) have developed the first practical B. mori multiple nucleopolyhedrovirus (BmMNPV) bacmid system directly applicable to protein expression in silkworms. This method provides rapid protein production in silkworms within 10 days, is free from biohazards, and thus will be a powerful tool as a future production factory of recombinant eukaryotic proteins because the bacmid does not require any baculovirus amplification step. Using this system, the green fluorescent protein was successfully expressed in silkworm larvae and pupae not only by infection with the recombinant virus but also by direct injection of its bacmid DNA. However, we found that the silkworm larvae were liquefied 5 days postinfection. Ohkawa et al. (1994) reported that BmMNPV also encodes v-cath-like cysteine protease, which has an extremely high homology (96.3\%) to Autographa californica multiple nucleopolyhedrovirus putative viral cysteine protease (Whitford et al., 1989). Suzuki et al. (1997) constructed BmMNPV virus lacking the cysteine protease gene and produced 
1 firefly luciferase and human growth factor in silkworms very efficiently because of the

2 markedly reduced degradation of these proteins.

3 In this study, we constructed a cysteine protease-deleted BmMNPV

4 (CPD-BmMNPV) bacmid using the lambda recombination system. The

$5 \quad$ CPD-BmMNPV bacmid provides rapid protein production in silkworms with less

6 degradation of recombinant eukaryotic proteins, showing improved expression of

$7 \quad \mathrm{GFP}_{\mathrm{uv}}-\beta 1,3-N$-acetylglucosaminyltransferase 2 (GGT2) fusion protein. These results

8 indicate that the CPD-BmMNPV bacmid is suitable for the future production of

9 recombinant eukaryotic proteins free from biohazards.

\section{Materials and Methods}

\subsection{Bacterial cells, plasmids and media} Escherichia coli DH10Bac was obtained from Invitrogen (Carlsbad, CA, USA). pKD3 and pKD46 were kind gifts from Dr. Mary K. B. Berlyn of Yale University. The vectors for BmMNPV and the wild-type viral BmMNPV DNA were obtained from Funakoshi Co. (Tokyo, Japan), and pENTER/D-TOPO and Gateway pDEST8 were purchased from Invitrogen. The $\mathrm{GFP}_{\mathrm{uv}}$ gene was obtained by PCR from pBlueBacHis2-GFP ${ }_{u v} / \beta 3$ GnT2 (Kato et al., 2004). The B. mori Bm5 cell line was

21 provided by Dr. K. S. Boo from the Insect Pathology Laboratory of Seoul National University. Sf900 II serum-free medium (Invitrogen) supplemented with 0.5\% 
1 antibiotic-antimycotic (Invitrogen) and 1\% FBS was used for B. mori Bm5 cell culture.

2 Supplements, if necessary, were added into the media at the following concentrations:

3 ampicillin, $100 \mu \mathrm{g} / \mathrm{mL}$; kanamycin, $50 \mu \mathrm{g} / \mathrm{mL}$; Bluo-gal (Invitrogen), $100 \mu \mathrm{g} / \mathrm{mL}$;

4 gentamycin, $7 \mu \mathrm{g} / \mathrm{mL}$; tetracycline, $10 \mu \mathrm{g} / \mathrm{mL}$ and isopropyl- $\beta$-D-thiogalactopyranoside,

$5 \quad 40 \mu \mathrm{g} / \mathrm{mL}$. The cysteine protease inhibitor

6 [trans-epoxysuccinyl-L-leucylamido-(4-guanidino) butane] was purchased from Wako

$7 \quad$ Pure Chem. Ind. Ltd. (Osaka, Japan).

9 2.2. Construction of CPD-BmMNPV bacmid in Fig. 1. A CPDcat fragment of $1.1 \mathrm{~kb}$ was obtained by PCR using the chloramphenicol

13 acetyltransferase gene of pKD3 using Bm98758Fcat and Bm99687Rcat (Table 1) containing $50 \mathrm{bp}$ sequences from start codon and stop codon of v-cath, respectively. The pKD3 template mixed in the PCR product was digested with Dpn I restriction enzyme.

Red recombinase plasmid pKD46 was transformed to DH10Bac competent cells containing BmMNPV bacmid DNA (Motohashi et al., 2005). The resulting transformed cells were grown on Luria-Bertani (LB) agar plates (Sambrook et al., 1989) containing kanamycin and ampicillin at $30^{\circ} \mathrm{C}$ overnight. Grown colonies were selected on SOB agar plates containing $0.2 \% \mathrm{~L}$-arabinose at $30^{\circ} \mathrm{C}$ overnight. Purified CPDcat fragment 
1 DNA and pKD46 using electroporation. Kanamycin- and chloramphenicol-resistant

2 colonies were selected on LB agar plates at $37^{\circ} \mathrm{C}$. After confirming the replacement of

3 the v-cath gene with the cat gene by PCR, the CPD-BmMNPV bacmid containing

4 deleted v-cath gene was isolated. Approximately $100 \mathrm{ng}$ of CPD-BmMNPV bacmid

5 DNA was transfected into Bm5 cells by lipofection. After incubation of the Bm5 cells

6 for $72 \mathrm{~h}$ at $27^{\circ} \mathrm{C}, v \mathrm{CPD}-\mathrm{BmMNPV}$ was harvested. The harvested recombinant virus was

7 diluted tenfold, and infected into Bm5 cells for amplification. The final amplified

8 recombinant virus was stored for infection into silkworm larvae.

\subsection{Infection with recombinant virus}

Bm5 cells were infected with $1 \times 10^{6} \mathrm{pfu} / \mathrm{mL} v \mathrm{CPD}-\mathrm{BmMNPV}$, and the infected

$13 \mathrm{Bm} 5$ cells were incubated for $72 \mathrm{~h}$ at $27^{\circ} \mathrm{C}$. The cell pellet was separated from $300 \mu \mathrm{L}$

14 of culture broth by centrifugation at $2,000 \mathrm{~g}$ for $15 \mathrm{~min}$. Three hundred microliters of 50

$15 \mathrm{mM}$ Tris- $\mathrm{HCl}$ containing $1 \%$ Triton $\mathrm{X}-100$ were added to the cell pellet and placed on

16 ice for $15 \mathrm{~min}$. The supernatant was assayed for intracellular protease activity. For

17 silkworm infection, fifth instar larvae (Ehime Sansyu Co. Ltd., Ehime, Japan) were used

18 for the expression of fusion protein. Twenty microliters of $1 \times 10^{6} \mathrm{pfu} / \mathrm{mL}$

vCPD-BmMNPV suspended in Sf900II serum-free medium were injected into the

20 dorsal region of the larvae using a syringe with 26-gauge beveled needle. Hemolymph

$21(100 \mu \mathrm{L})$ was harvested $4 \mathrm{~d}$ postinjection and added to $100 \mu \mathrm{L}$ of $50 \mathrm{mM}$ Tris- $\mathrm{HCl}$ 
1 containing $1 \%$ Triton X-100 for protease activity assay.

2

2.4. Construction of $\downarrow C P D-B m M N P V$ expressing GGT2 fusion protein

4

5

CPD-BmMNPV bacmid DNA was transformed into DH10Bac competent cells

6 containing pHelper, which encodes a transposase and confers resistance to tetracycline.

7 The DH10Bac/CPD-BmMNPV bacmid/pHelper cells were screened on LB agar plates

8 containing kanamycin and tetracycline. The selected colonies were cultured to prepare

9 fresh competent cells for the Bac-to-Bac system.

To enable the introduction of a signal-peptide-coding region, the GGT2 fusion fragment of pBlueBacHis2-GGT2 (Kato et al., 2003) was amplified using a PCR primer set (Table 1). This PCR product, including the signal sequences, was cloned into the Gateway cloning vector pENTR/D-TOPO (Invitrogen) according to the protocol

14 provided for the TOPO cloning system. The isolated plasmid was designated pENTR/D/bx-GGT2, in which the GGT2 fusion gene fused with the silkworm

16 bombyxin signal sequence (Adachi et al., 1989). Fifty micrograms of pDEST8/bx-GGT2 DNA were transformed into $100 \mu \mathrm{L}$ of DH10Bac/CPD-BmMNPV bacmid/pHelper competent cells. The transformed cells were added to $500 \mu \mathrm{L}$ of SOC and incubated at $37^{\circ} \mathrm{C}$ for $1 \mathrm{~h}$, and then were cultured on LB agar plates containing gentamycin, kanamycin, tetracycline, isopropyl- $\beta$-D-thiogalactopyranoside, and 
1 colonies were inoculated into LB medium containing gentamycin, kanamycin, and

2 tetracycline and cultured at $37^{\circ} \mathrm{C}$ with agitation at $150 \mathrm{rpm}$ overnight. $\mathrm{CPD}-\mathrm{BmMNPV}$

$3 \mathrm{bacmid} / \mathrm{bx}-\mathrm{GGT} 2$ was isolated and confirmed by PCR using the bx-GGT2 and reverse

4 primers (Table 1).

5

6 2.5. Expression of GGT2 fusion protein in silkworm larvae

7

Approximately 200 ng of CPD-BmMNPV bacmid/bx-GGT2 DNA were injected

9 into fifth instar larvae in a similar way as described earlier. The fusion protein contained in the hemolymph was stocked for $\beta 3 \mathrm{GnT}$ and cysteine protease assays.

2.6. $\beta 3 G n T$ and cysteine protease assay

$\beta 3 \mathrm{GnT}$ activity assay was carried out according to the protocol described by Kato et al. (2004).

Cysteine protease assay at neutral $\mathrm{pH}$ was performed as described by Hom et al. (2002). First, the sample ( $83 \mu \mathrm{L})$ was added to $583 \mu \mathrm{L}$ of $0.2 \%$ azocasein in $50 \mathrm{mM}$ sodium phosphate buffer ( $\mathrm{pH} 7.2$ ). The mixture was incubated at $37^{\circ} \mathrm{C}$ for $4 \mathrm{~h}$, followed by the addition of $27 \mu \mathrm{L}$ of $100 \%$ TCA to stop the reaction. Hemolymph samples were centrifuged at $25,000 \mathrm{~g}$ for $5 \mathrm{~min}$, and $9 \mathrm{M}$ urea $(333 \mu \mathrm{L})$ was added to the supernatant

20 before measuring the absorbance at $410 \mathrm{~nm}$. One unit of cysteine protease activity was defined as the amount of enzyme capable of increasing the absorbance by 1 at $410 \mathrm{~nm}$ 
1 within $1 \mathrm{~h}$.

2

\subsection{SDS-PAGE}

4

The protein contained in the hemolymph was subjected to SDS-PAGE on a $12 \%$

6 polyacrylamide gel using the Mini-protean II system (Bio-Rad, Hercules, CA). To

7 detect directly the molecular band of a specific GGT2 protein on SDS-PAGE gel using

8 Molecular Imager FX (Bio-Rad), the samples were only mixed with sample buffer

9 without boiling. In this case, the molecular mass shifted to a slightly lower value compared to boiled samples on SDS-PAGE gel (Aoki et. al., 1996).

\section{Results}

\subsection{Construction of CPD-BmMNPV bacmid}

We prepared the CPDcat fragment, which consists of $50 \mathrm{bp}$ fragments of the 5'-

(3-52) and 3'- (884-933) regions in v-cath and complete cat gene from pKD3, by PCR with Bm98758Fcat and Bm99687Rcat (Table 1) containing 50 bp sequences from start codon and stop codon of v-cath, respectively. To construct v-cath-deleted bacmid, CPD-BmMNPV bacmid, by homologous recombination with CPDcat fragment, we used the Gam-producing plasmid pKD46 in which the bet, exo, and gam genes were under the control of an arabinose operon (Datsenko and Wanner, 2000). The Gam 
1 prevents RecBCD-promoted digestion of $\lambda$ phage DNA, so that Exo and Bet can gain

2 access to DNA ends to promote recombination (Murphy, 1998). This homologous

3 recombination was useful for the construction of the CPD-BmMNPV bacmid.

4 Deletion of the v-cath gene was confirmed by PCR using the bacmid extracted

5 from kanamycin- and chloramphenicol-resistant colonies as a template. The v-cath gene

6 fragment, including the promoter and signal peptide sequences, was approximately 1.3

$7 \mathrm{~kb}$, but the $\mathrm{v}$-cath deleted fragment containing cat gene was approximately $1.5 \mathrm{~kb}$ as

8 was expected (Fig. 2). Elimination of pKD46 occurred because replicon is temperature

$9 \quad$ sensitive and cannot work at $37^{\circ} \mathrm{C}$.

3.2. Protease activity in Bm5 cells and silkworm larvae using vCPD-BmMNPV bacmid

When $v$ BmMNPV and $v$ CPD-BmMNPV were infected into Bm5 cells, their intracellular protease activities were measured at $72 \mathrm{~h}$ postinjection. The protease activities of $v$ BmMNPV- and $v C P D-B m M N P V$-infected Bm5 cells were 0.34 and 0.02 $\mathrm{U} / \mathrm{mL}$, respectively (Fig. 3). The protease activity of $v$ CPD-BmMNPV-infected Bm5 cells was the same level as that of $v B m M N P V$-infected Bm5 cells with the addition of cysteine protease inhibitor, [trans-epoxysuccinyl-L-leucylamido-(4-guanidino) butane, suggesting that the decrease in protease activity of $v$ CPD-BmMNPV-infected Bm5 cells was due to v-cath deletion.

Figure 4A shows a comparison of the liquefaction times of the silkworm larvae. 
1 The larvae infected with $v$ BmMNPV were liquified at $5 \mathrm{~d}$ postinjection, whereas the

2 larvae infected with $v$ CPD-BmMNPV almost retained their normal white skin and

3 neither melanized nor melted at $5 \mathrm{~d}$ postinjection. For silkworm larvae infected with

$4 \quad$ CPD-BmMNPV, the protease activity of the hemolymph was $0.32 \mathrm{U} / \mathrm{mL}$ (Fig. 4B),

$5 \quad$ which was only $15 \%$ in comparison with that of $v \operatorname{BmMNPV}(2.03 \mathrm{U} / \mathrm{mL})$. For Bm5

6 cells and silkworm larvae, $v$ CPD-BmMNPV suppressed protease activity by $94 \%$ and

$7 \quad 85 \%$, respectively, compared with Bm5 cells and silkworm larvae infected with

8 BmMNPV, because of the deletion of cysteine protease in the BmMNPV bacmid.

3.3 Protease activity and $\beta 3 G n T 2$ expression in silkworms using

CPD-BmMNPV/bx-GGT2 bacmid

Protease activity and GGT2 fusion protein expression in the

CPD-BmMNPV/bx-GGT2 bacmid-injected silkworm larvae are shown in Figs. 5 and 6, respectively. When the BmMNPV bacmid was injected, the silkworm larvae melted at 7

d postinjection, but the silkworm larvae injected with CPD-BmMNPV/bx-GGT2 bacmid remained intact and showed no symptoms of liquefaction (Fig. 5A). The protease activities of the silkworm larvae hemolymphs infected with the 
1 observed in the hemolymph infected with the CPD-BmMNPV/bx-GGT2 bacmid, which

2 is the same result shown in Fig. 3. The protease activity of mock was similar to that of

3 the hemolymph infected with the CPD-BmMNPV/bx-GGT2 bacmid. The difference in

4 the protease activity of the hemolymphs between the $v B m M N P V$ and BmMNPV

5 bacmids may be due to the degree of infectivity.

6 The maximum $\beta 3 \mathrm{GnT}$ activity in the hemolymph of the silkworm larvae injected

7 with the CPD-BmMNPV/bx-GGT2 bacmid was $39.3 \mathrm{mU} / \mathrm{mL}$ at $4 \mathrm{~d}$ postinjection,

8 whereas that in the hemolymph of the silkworm larvae injected with the

9 BmMNPV/bx-GGT2 bacmid was $29.7 \mathrm{mU} / \mathrm{mL}$ (Fig. 6A). The low $\beta 3 \mathrm{GnT}$ activity in 10 the hemolymph injected with the BmMNPV/bx-GGT2 bacmid might be caused by the

11 degradation of $\beta 3 \mathrm{GnT} 2$ by proteases. The full-length fusion protein disappeared in SDS-PAGE gels at $4 \mathrm{~d}$ postinjection due to proteolytic degradation, and low molecular

13 bands appeared (Fig. 6B). However, the fusion protein band in the hemolymph injected with the CPD-BmMNPV/bx-GGT2 bacmid had thickened at $4 \mathrm{~d}$ postinjection without degradation.

\section{Discussion}

Silkworms or pupae are one of the most suitable systems for the efficient production of eukaryotic proteins because of their more extensive expression of functional proteins than insect cells or human-oriented cultured cells. However, until now, silkworms have 
1 been troublesome for protein expression because of the requirement of a long

2 recombination time in baculovirus and because several rounds of virus purification and

3 amplification of viruses in insect cells are required to obtain high titer virus

4 concentrations. The development of the practical BmMNPV bacmid system directly

5 applicable to protein expression in silkworms solved these problems (Motohashi et al.,

$62005)$.

$7 \quad$ Many studies (Kaba et al., 2004; Lee et al., 2006; Wang et al., 2005) have indicated

8 that foreign gene products in silkworm larvae are frequently degraded owing to cysteine

9 proteases originating from the BmMNPV gene. This is a serious problem in terms of the mass production of proteins of interest in silkworm larvae. To overcome this problem,

11 we constructed a practical CPD-BmMNPV bacmid, in which the v-cath gene was replaced with the chloramphenicol acetyltransferase gene. We observed that the protease

13 activities of Bm5 cells and silkworm larvae infected with this vCPD-BmMNPV were

14 reduced by $94 \%$ and $85 \%$, respectively. The larvae infected with $v$ CPD-BmMNPV maintained their normal white skin without liquefaction even at $5 \mathrm{~d}$ postinjection. Compared with the liquefaction induced by virus infection, the liquefaction of the silkworm larvae in this study was delayed by $2 \mathrm{~d}$. The expression level of the fusion protein was also higher than that by injection with the conventional BmMNPV bacmid. This shows that the CPD-BmMNPV bacmid was an efficient tool for expressing 20 eukaryotic genes in the hemolymph of the silkworm larvae. 
1 cost of production, as silkworms are easy to treat and have a high safety level in terms

2 of biohazards. We believe that the CPD-BmMNPV bacmid can be utilized as a very

3 effective expression vector for the stable production of useful foreign proteins,

4 particularly proteins susceptible to cysteine protease. Furthermore, for production of

5 secretory proteins in silkworm larvae, it is important to collect hemolymph before the

6 degradation of target-gene products begins. Using this CPD-BmMNPV bacmid as an

7 expression vector, undegraded proteins of interest might be harvested and degradation

8 of proteins of interest during purification from hemolymph might be prevented.

Acknowledgements

This work was supported by the Program of Basic Research Activities for

Innovative Biosciences (PROBRAIN), Japan. We are very grateful to Dr. Mary K. B.

14 Berlyn of Yale University for kindly providing plasmids pKD3 and pKD46, to

15 Professors T. Murata and T. Usui in the Biochemistry Lab. of Shizuoka University for

16 kindly providing substrate of $\beta 3 \mathrm{GnT}$ assay, and to Professor K. S. Boo in the Insect

17 Pathology Lab. of Seoul National University for kindly providing B. mori cells.

References

Adachi, T., Takiya, S., Suzuki, Y., Iwami, M., Kawakami, A., Takahashi, S.Y., Ishizaki, H., Nagasawa, H., Suzuki, A., 1989. cDNA structure and expression of Bombyxin, 
an Insulin-like brain secretory peptide of the silkmoth Bombyx mori. J. Biol. Chem. $264,7681-7685$.

Aoki, T., Takahashi, Y., Koch, K. S., Leffert, H. L., Watabe, H., 1996. Construction of a fusion protein between protein A and green fluorescent protein and its application to Western blotting. FEBS Lett. 384, 193-197.

Datsenko, K. A., Wanner, B. L., 2000. One-step inactivation of chromosomal genes in Escherichia coli K-12 using PCR products. Proc. Natl. Acad. Sci. 97, 6640-6645.

Davis, T.R., Trotter, M., Granados, R.R., Wood, H.A., 1992. Baculovirus expression of alkaline phosphatase as reporter gene for evaluation of production, glycosylation and secretion. Bio/Technology 13, 592-596.

Hericourt, F., Blanc, S., Redeker, V., Jupin, I., 2000. Evidence for phosphorylation and ubiquitinylation of the turnip yellow mosaic virus RNA-dependent RNA polymerase domain expressed in a baculovirus-insect cell system. Biochem. J. 349, 417-425.

Hom, L.G., Ohkawa, T., Trudeau, D., Volkman, L.E., 2002. Autographa californica M nucleopolyhedrovirus proV-CATH is activated during infected cell death. Virol. 296, 212-218.

James, D.C., Freedman, R.B., Hoare, M., Ogonash, O.W., Rooney, B.C., Larionov, O.A., Dobrovolsky, V.N., Lagutin, O.V., Jenkins, N., 1995. N,N-glycosylation of recombinant human interferon-produced in different animal expression system. Bio/Technology 13, 592-596.

Kaba, S.A., Salcedo, A.M., Wafula, P.O., Vlak, J.M., van Oers, M.M., 2004. 
Development of a chitinase and v-cathepsin negative bacmid for improved integrity of secreted recombinant proteins. J. Virol. Methods 122, 113-118.

3 Kato, T., Murata, T., Usui, T., Park, E.Y., 2003. Improvement of GFP ${ }_{u v}-\beta 3$ GnT2 fusion protein production by suppressing protease in baculovirus expression system. Biosci. Biotechnol. Biochem. 67, 2288-2395.

6 Kato, T., Murata, T., Usui, T., Park, E. Y., 2004. Efficient production of human

$\beta-1,3-N$-acetylglucosaminyltransferase-2 fused with green fluorescence protein in insect cell. Biochem. Eng. J. 19, 15-23.

Lee, K.S., Je, Y.H., Woo, S.D., Sohn, H.D., Jin, B.R., 2006. Production of a cellulose in silkworm larvae using a recombinant Bombys mori nucleopolyhedrovirus lacking the virus-encoded chitinase and cathepsin genes. Biotechnol. Lett. 28, 645-650.

Monsma, S.A., Scott, M.D., 1997. Bacvector-3000: An engineered baculovirus designed for greater protein stability. inNovations 7, 16-19.

Motohashi, T., Shimojima, T., Fukagawa, T., Maenaka, K., Park E.Y., 2005. Efficient

large-scale protein production of larvae and pupae of silkworm by Bombyx mori nuclear polyhedrosis virus (BmNPV) bacmid system. Biochem. Biophys. Res.

$7 \quad$ Comm. 326, 564-569.

8 Murphy, K. C., 1998. Use of bacteriophage $\lambda$ recombination function to promote gene 9 replacement in Escherichia coli. J. Bacetriol. 180, 2603-2071.

20 Ohkawa, T., Majima, K., Maeda, S., 1994. A cysteine protease encoded by the 21 baculovirus Bombyx mori nuclear polyhedrosis virus. J. Viol. 68, 6619-6625. 
1 Pham, M., Naggie, S., Wier, M., Cha, H., Bentley, W.E., 1999. Human interleukin-2

2 production in insect cell (Trichoplusia ni) larvae: effects and partial control of

3 proteolysis. Biotechnol. Bioeng. 62, 175-182.

Pyle, L.E., Barton, B., Fujiwara, Y., Mitchell, A., Fidge, N., 1995. Secretion of biological active human proapolipoprotein A-I a baculovirus-insect cell system: protection from degradation by protease inhibitor. J. Lipid Res. 36, 2355-2361.

Sambrook, J., Fritsch, E. F., Maniatis, T., 1989. Molecular cloning: a laboratory manual, $2^{\text {nd }}$ ed. Cold Spring Harbor Laboratory Press, Cold Spring Harbor, N.Y.

Slack, J.M., Kuzio, J., Faulkner, P., 1995. Characterization of v-cath, a cathepsin L-like protease expressed by the baculovirus Autographa californica multiple nuclear polyhedrosis virus. J. Gen. Viol. 76, 1091-1098.

Suzuki, T., Kanaya, T., Okazaki, H., Ogawa, K., Usami, A., Watanabe, H., Kadono-Okuda, K., Yamakawa, M., Sato, H., Mori, H., Takahashi, S., Oda, K., 1997. Efficient protein production using a Bombyx mori nuclear polyhedrosis virus lacking the cysteine proteinase gene. J. Gen. Virol. 78, 3073-3080.

Wang, F., Zhang, C.X., Kumar, V.S., Wu, X.F., 2005. Influences of chitinase gene deletion from BmNPV on the cell lysis and host liquification. Archiv. Virol. 150, 981-990.

Whitford, M. Stewart, S., Kuzio, J., Faulkner, P., 1989. Identification and sequence analysis of a gene encoding gp64, an abundant envelope glycoprotein of the baculovirus Autographa californica nuclear polyhedrosis virus. J. Virol. 63, 

1393-1399.

2 Yamada, K., Nakajima, Y., Natori, S., 1990. Production of recombinant sarcotoxin IA in 3 Bombyx mori cells. Biochem. J. 272, 633-636.

4 


\section{Legends for figures}

2

3 Fig. 1. Schematic outline for construction of CPD-BmMNPV bacmid. A CPDcat PCR fragment was obtained from pKD3. DH10Bac cells that were ampicillin- and kanamycin-resistant were selected and then incubated on an LB plate with $0.2 \%$ L-arabinose at $30^{\circ} \mathrm{C}$ to induce Gam, Bet, and Exo from pKD46. The CPDcat PCR fragment was introduced to DH10Bac cells containing BmMNPV DNA and pKD46 by electroporation. The CPDcat PCR fragment and BmMNPV bacmid DNA recombined homologously. Chloramphenicol- and kanamycin-resistant cells were selected under conditions of incubation overnight at $37^{\circ} \mathrm{C}$ and designated CPD-BmMNPV bacmid.

Fig. 2. Confirmation of v-cath gene deletion by PCR. Lane M denotes DNA marker. Lanes 1 and 2 denote PCR products from BmMNPV bacmid and CPD-BmMNPV bacmid, respectively. The v-cath gene fragment was approximately $1.3 \mathrm{~kb}$ because of including the promoter and signal peptides, but the v-cath gene fragment containing cat gene was approximately $1.5 \mathrm{~kb}$.

Fig. 3. Protease activity in Bm5 cells infected with BmMNPV and CPD-BmMNPV viruses (A) and hemolymph fluid of silkworm larvae (B), respectively.

Fig. 4. Comparison of larval liquefaction (A) and protease activity (B) infected with BmMNPV and CPD-BmMNPV viruses. The left column in (A) indicates postinjection time (d). 
1 Fig. 5. Comparison of larval liquefaction (A) and protease activity (B) injected with

2 BmMNPV and CPD-BmMNPV bacmids. The left column indicates postinjection

3 time (d). Protease activity in the hemolymph fluid of mock, injected with BmMNPV

$4 \quad$ and CPD-BmMNPV bacmids at $6 \mathrm{~d}$ postinjection was measured.

$5 \quad$ Fig. 6. $\beta 3 \mathrm{GnT}$ activity (A) and $\mathrm{GFP}_{\mathrm{uv}}$ fluorescent analysis of GGT2 fusion protein (B)

6 in the hemolymph of silkworms injected with BmMNPV/bx-GGT2 (white bars) and

$7 \quad$ CPD-BmMNPV/bx-GGT2 bacmids (black bars). Protein bands in (B) were detected

8 by $\mathrm{GFP}_{\mathrm{uv}}$ fluorescence. Bars denote standard deviations $(\mathrm{n}=3)$. 


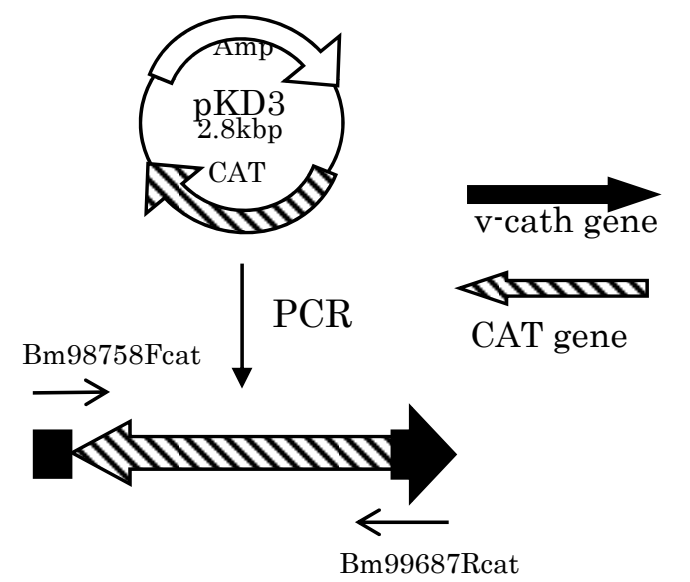

CPDcat PCR fragment (1.1kbp)
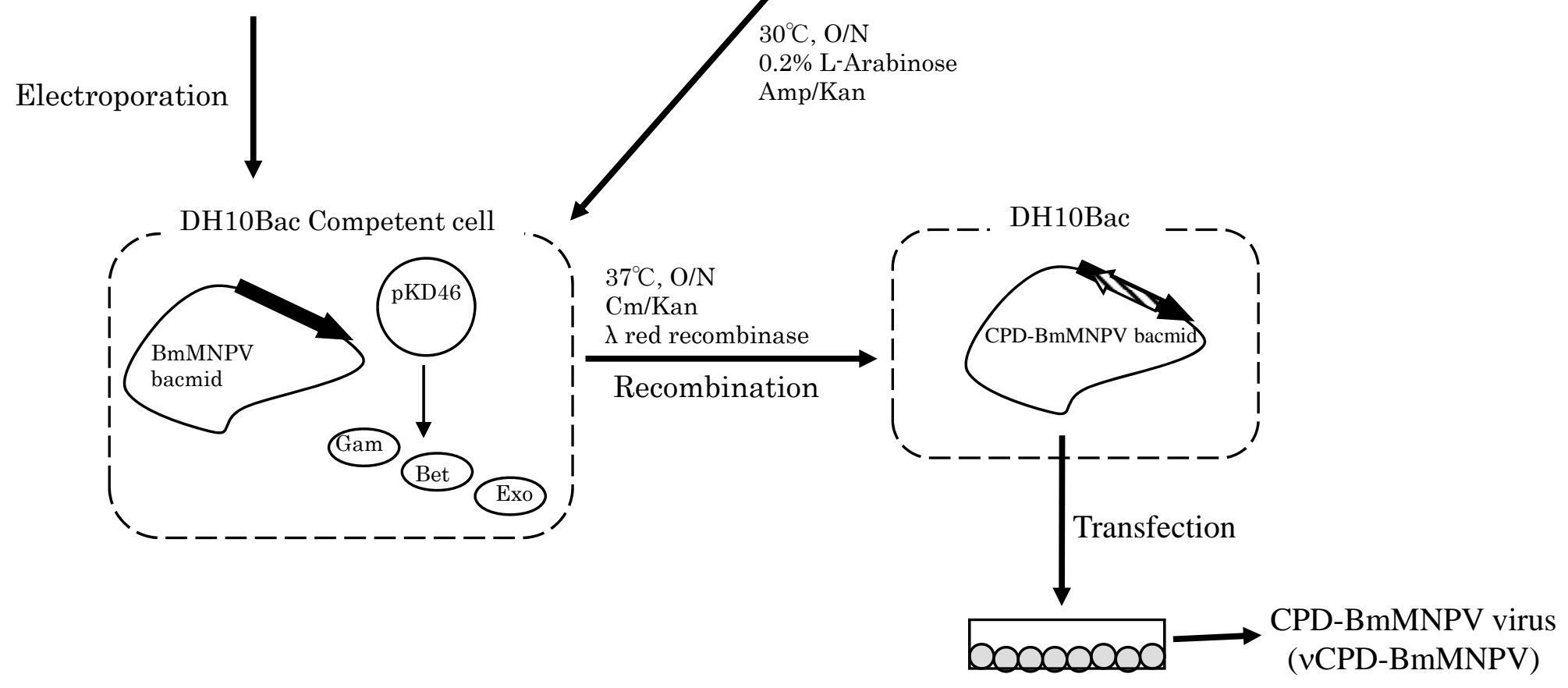


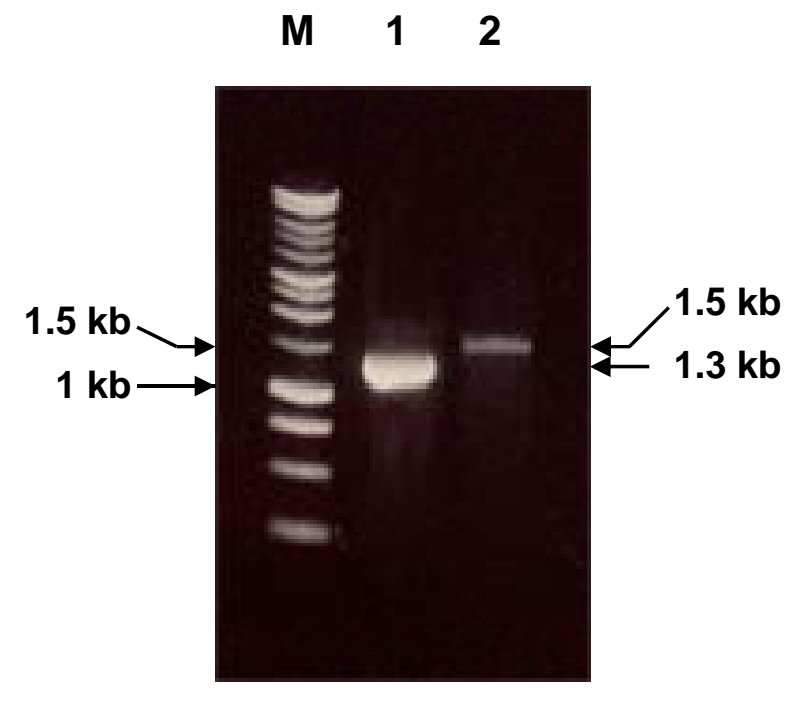




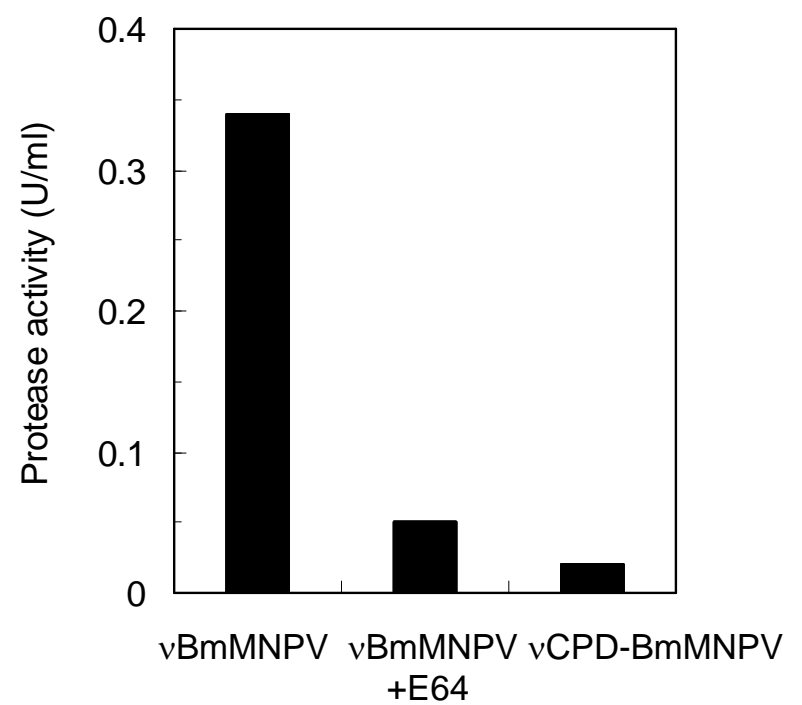




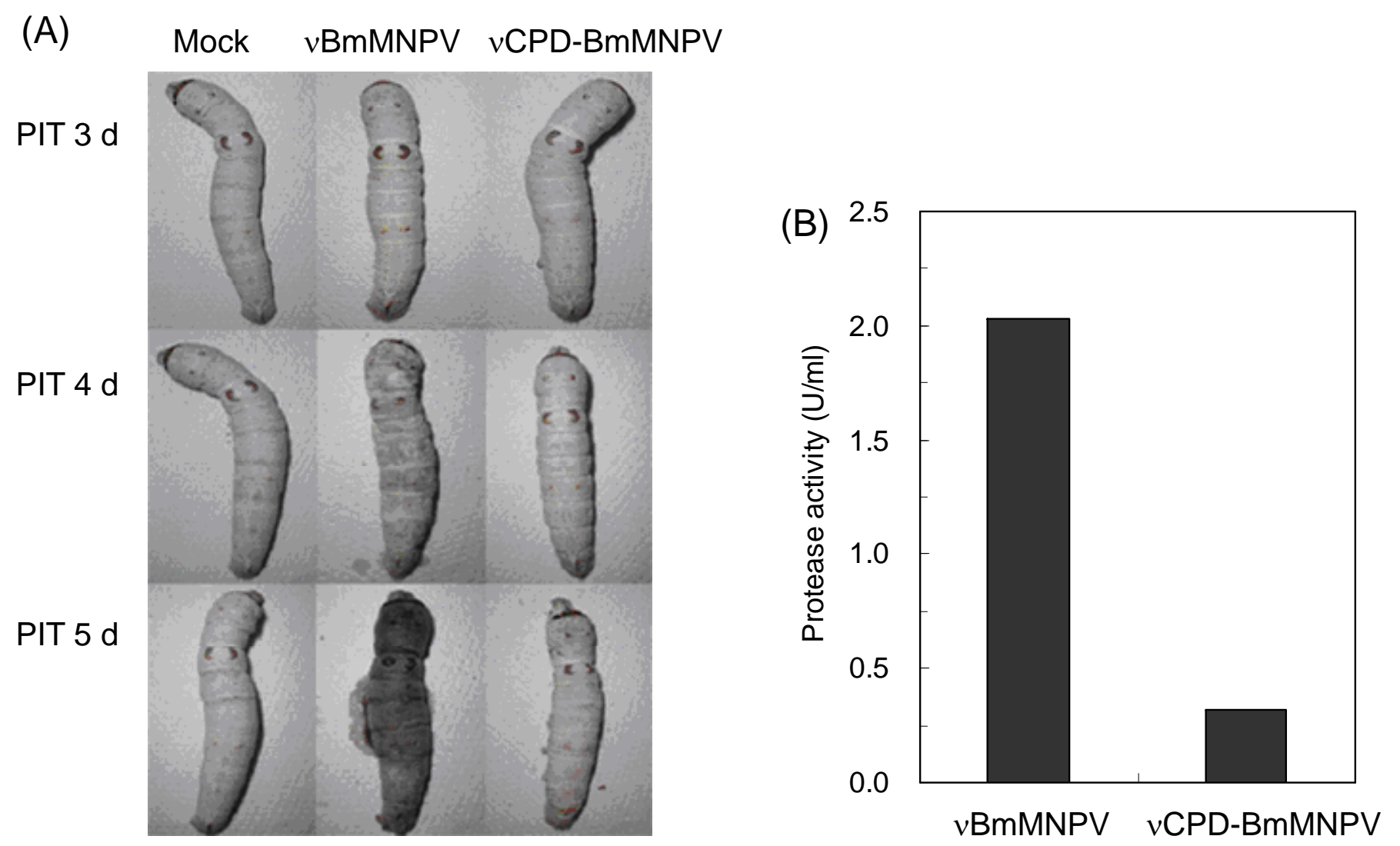


(A) BmMNPV CDP-BmMNPV bacmid bacmid

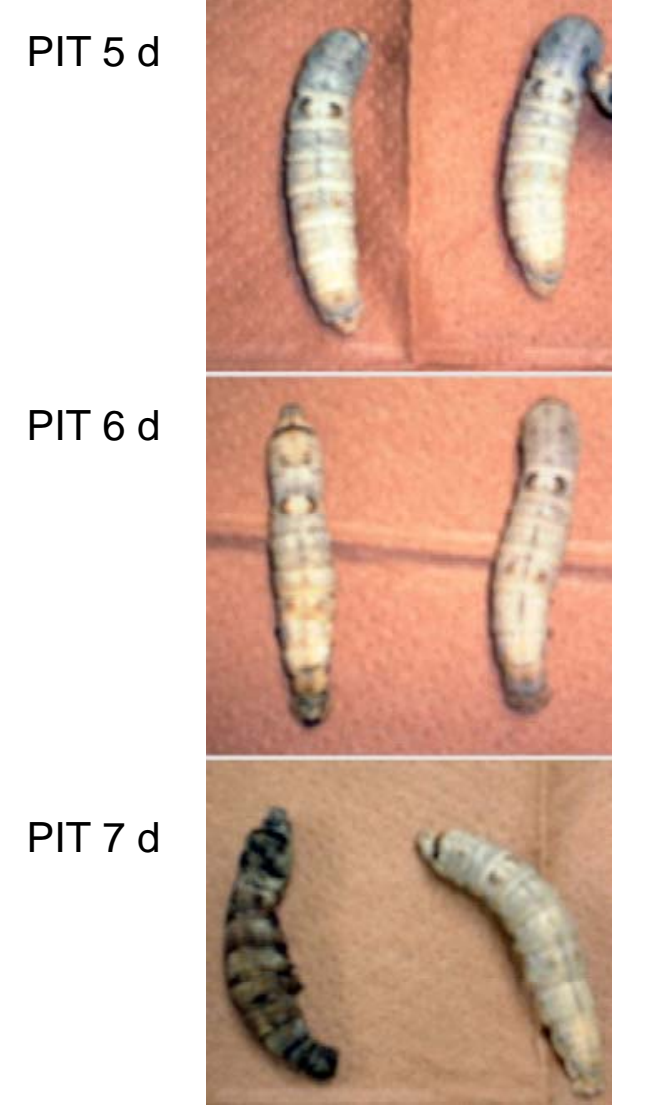

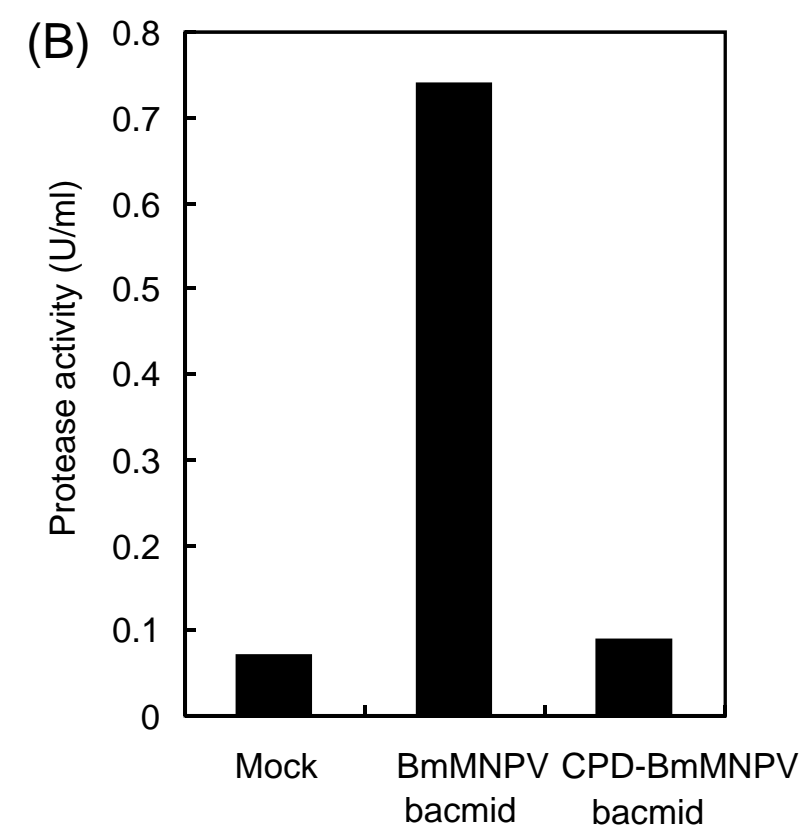


(A)

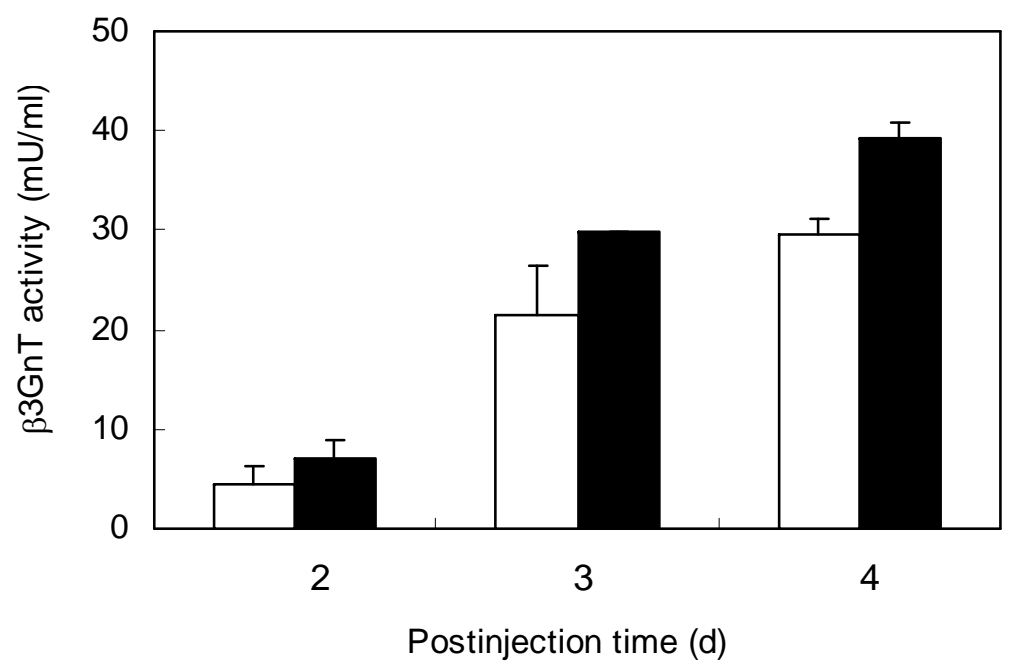

(B)

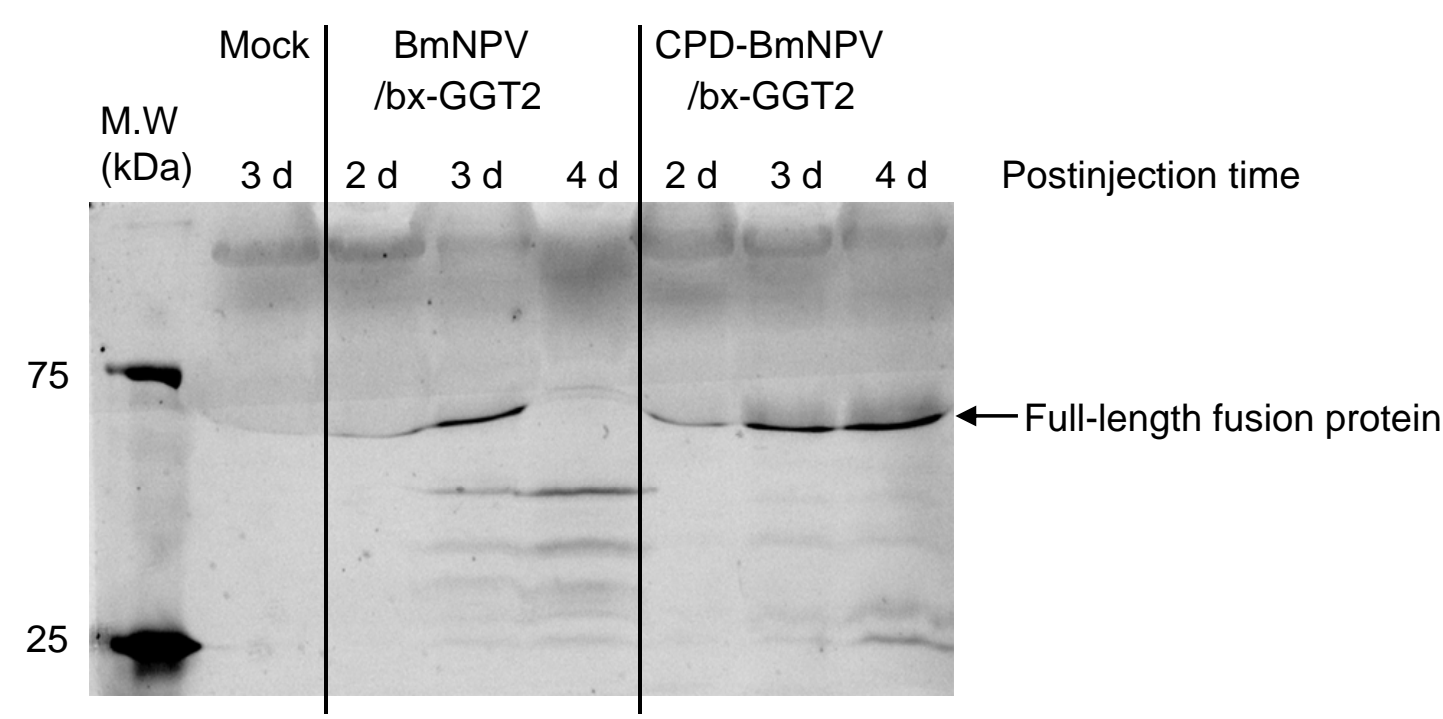


Table 1

Primers used to prepare CPDcat fragment and signal sequence

\begin{tabular}{|c|c|}
\hline Primer-name & Sequence $\left(5^{\prime} \rightarrow 3^{\prime}\right)$ \\
\hline Bm98758Fcat & $\frac{\text { GAACAAAATTTTGTTTTATTTGTTTGTGTACGCCGTTGTAAAGAGCGCGG }}{\text { v-cath }} \frac{\text { TTGTGTAGGCTGGAGCTGCT }}{\text { chloramphenicol acetyltransferase }}$ \\
\hline Bm99687Rcat & $\frac{\text { TAATAAATGACTGCAGTAGACGCAAGTTCGTTTCTCATACCACAAGCGT }}{\text { v-cath }} \frac{\text { TCCATATGAATATCCTCCTTA }}{\text { chloramphenicol acetyltransferase }}$ \\
\hline & CACCATGAAGATACTCCTTGCTATTGCATTAATGTTGTCAACAGTAATGTGGGTGTCAACACAACCGCGG \\
\hline $\mathrm{bx}-\mathrm{GFP}_{\mathrm{uv}}{ }^{-}$ & Signal sequence 1 \\
\hline$\beta 3 \mathrm{GnT} 2$ & $\frac{\text { GGTTCTCATCATC }}{\text { GFPuv- } \beta 3 \text { GnT2 }}$ \\
\hline Reverse primer & CGGAATTCTGAAGGGTTTAGAGGCCCTCAAATGGG \\
\hline
\end{tabular}

